\title{
Quimioterapia Neoadjuvante em Câncer Localmente Avançado do Colo do Útero
}

\author{
Neoadjuvant Chemotherapy in Locally Advanced Cancer of the Cervix
}

Eduardo Schünemann $\mathrm{Jr}^{1}$, Cícero de Andrade Urban ${ }^{1}$, Vinícius Milani Budel ${ }^{2}$

\section{RESUMO}

\begin{abstract}
Objetivos: avaliar a quimioterapia neoadjuvante no câncer localmente avançado do colo uterino, por meio da sua aceitabilidade, tolerabilidade, toxicidade, taxa de complicações cirúrgicas, taxa de resposta, taxa de operabilidade e sobrevida em 5 anos.

Métodos: foram incluidas 60 mulheres com câncer do colo uterino localmente avançado (IIB e IIIB), submetidas à quimioterapia neoadjuvante com doxorrubicina-bleomicina-cisplatina. Aquelas que se tornaram operáveis após a quimioterapia foram submetidas à cirurgia de Wertheim-Meigs, seguida de radioterapia pélvica complementar. Nas pacientes em que a cirurgia não foi possivel após a quimioterapia, realizou-se radioterapia.

Resultados: o seguimento médio foi de 108 meses. A taxa de resposta global à quimioterapia foi de $80 \%$, sendo $100 \%$ para o estádio IIB e $60 \%$ para o estádio IIIB. A porcentagem de pacientes operadas, após a quimioterapia foi de 65\%. A sobrevida global em 5 anos para todo o grupo foi 62\%. No grupo operado ( $n=34)$, a sobrevida global foi de 82,14\%, independentemente do estádio inicial. No grupo não operado ( $n=18)$, a sobrevida em 5 anos foi $16,67 \%$.

Conclusões: A quimioterapia neoadjuvante com doxorrubicina-bleomicina-cisplatina no câncer do colo uterino localmente avançado é segura, com baixo indice de complicações e permitiu uma alta taxa de operabilidade.
\end{abstract}

PALAVRAS-CHAVE: Colo do útero: câncer. Quimioterapia neoadjuvante. Câncer: recorrência.

\section{Introdução}

O câncer de colo uterino é a segunda neoplasia mais freqüente no sexo feminino em todo o mundo. Estima-se que a incidência mundial esteja em torno de 500.000 novos casos/ano, sendo que $80 \%$ dos casos invasivos são diagnosticados em países em desenvolvimento ${ }^{1}$. No Brasil, a sua incidência está em primeiro lugar entre todos os tumores do aparelho genital feminino.

Apesar do grande avanço técnico ocorrido durante os últimos 40 anos, não houve mudanças

\footnotetext{
1 Serviço de Oncologia Ginecológica do Hospital Nossa Senhora das Graças

${ }^{2}$ Departamento de Tocoginecologia do Hospital de Clínicas da Universidade Federal do Paraná Correspondência:

Eduardo Schünemann Jr

Rua Rosa Saporski 320 - Mercês

80810-120 - Curitiba - PR

Fone/fax: (41) 335-3300

e-mail: lauraschu@hotmail.com
}

significativas em relação à sobrevida em 5 anos, quando analisado cada estádio em separado. No estádio I, a média de sobrevida em 5 anos varia entre 70 e $90 \%$; no estádio II, entre 40 e $60 \%$; no estádio III, entre 20 e $40 \%$ e, no estádio IV, entre zero e $20 \%^{2}$.

Os resultados insatisfatórios nos tumores avançados levaram à busca de novas estratégias terapêuticas e, entre elas, o uso da quimioterapia (QT). Nos últimos anos, os resultados de vários ensaios clínicos, principalmente empregando a QT nos tumores de colo uterino localmente avançados, trouxeram novas perspectivas para este grupo de pacientes ${ }^{3}$. Entretanto, o papel da cirurgia após a QT não foi adequadamente testado na maior parte destes estudos.

A pesquisa aqui apresentada fundamentouse em um estudo preliminar, realizado em 1985, que avaliou a resposta à QT neoadjuvante em câncer localmente avançado do colo uterino ${ }^{3}$. Neste estudo, 15 pacientes com estádios IIB e IIIB foram 
submetidas a QT, seguida de cirurgia e/ou radioterapia. Obteve-se assim $80 \%$ de resposta global, sendo $20 \%$ de resposta completa e $60 \%$ de resposta parcial. Deste total, procedeu-se ao tratamento cirúrgico em 10 (66\%) pacientes ${ }^{4}$.

Em 1987, a partir destes resultados favoráveis, iniciou-se este estudo. Foram selecionadas 60 pacientes com câncer do colo uterino localmente avançado, submetidas, seqüencialmente, à QT neoadjuvante com doxorrubicina-bleomicinacisplatina, cirurgia (quando possível) e radioterapia. Os resultados obtidos serão aqui apresentados e discutidos.

\section{Pacientes e Métodos}

O desenho deste estudo é de um ensaio clínico prospectivo e descritivo, para o qual foram recrutadas 60 pacientes (30 IIB e 30 IIIB), com média de idade de 48 anos, durante o período compreendido entre 1987 e 1995. As principais características das pacientes estão na Tabela 1 . O estudo recebeu aprovação prévia do Comitê de Ética do Hospital Nossa Senhora das Graças (Curitiba) e estava dentro das normas de pesquisa vigentes à época.

Tabela 1 - Perfil das 52 pacientes submetidas a quimioterapia neoadjuvante em câncer de colo uterino localmente avançado.

\begin{tabular}{lcc}
\hline & Estádio IIB & Estádio IIIB \\
\hline Número de pacientes & 27 & 25 \\
Média de idade & 47 & 49 \\
$\quad$ < de 30 anos & 1 & 0 \\
30 a 59 anos & 20 & 21 \\
$\quad$ > de 60 anos & 6 & 4 \\
Tipo histológico - CEC & 27 & 25 \\
Performance clínica 0 e 1 & 27 & 25 \\
\hline
\end{tabular}

CEC - carcinoma espinocelular

Os critérios de inclusão foram: câncer do colo uterino, estadiado segundo a FIGO (versão 1987) em IIB ou IIIB; tipo histológico - carcinoma espinocelular; assinatura do termo de consentimento informado; apresentar performance status entre 0 e 1 , conforme classificação do "Swiss Cooperative Group"; provas de funções hepática, renal, cardíaca, pulmonar e hematológica dentro dos limites normais; a idade máxima era de 67 anos e não deveria ter sido submetida anteriormente a tratamento oncológico. Foram excluídas as pacientes que não cumpriram as orientações do protocolo ou que perderam seguimento, as que recebiam tratamento oncológico concomitante para outro tipo de câncer ou que não puderam ou não desejaram continuar a receber o esquema quimioterápico.

O estadiamento foi realizado através do exame especular e do toque bimanual combinado, vaginal e retal. O tamanho do tumor no colo era medido diretamente em centímetros e a extensão parametrial foi avaliada pelo toque retal. O exame ginecológico foi realizado por três profissionais médicos, pertencentes ao Serviço de Oncoginecologia do Hospital Nossa Senhora das Graças e, somente quando houve concordância quanto ao estadiamento clínico, a paciente foi incluída no protocolo. Em casos de discordância sobre o estadiamento, um novo exame foi realizado, sob narcose, e, persistindo a discordância, a paciente era excluída da seleção.

Cada paciente foi submetida aos ciclos de QT, contendo três drogas, conforme o esquema: doxorrubicina - $50 \mathrm{mg} / \mathrm{m}^{2}$ dia 1 , cisplatina - 75 $\mathrm{mg} / \mathrm{m}^{2}$ dia 1 e bleomicina $-15 \mathrm{mg} / \mathrm{m}^{2}$ dia 1 , no dia 3 , a cada 3 ou 4 semanas. Foram aplicados no mínimo 2 ciclos e, no máximo, 4 ciclos, até a avaliação final da resposta. Cada ciclo de QT foi repetido somente se o número de leucócitos por $\mathrm{mm}^{3}$ estivesse acima de 3.000 e o de plaquetas acima de 100.000. Na proposta do estudo, quando a creatinina atingisse mais que $1,6 \mathrm{mg} / \mathrm{L}$, ou quando a paciente apresentasse manifestações clínicas, tais como dispnéia, taquicardia ou alterações no ECG ou na radiografia de tórax, a QT era suspensa.

O exame ginecológico era realizado após cada ciclo de QT, com mensuração direta do tamanho tumoral no colo uterino e avaliação parametrial por meio do toque retal. Nos casos em que houvesse redução tumoral a partir do segundo ou terceiro ciclos, tornando o tumor operável, a QT era suspensa e a paciente encaminhada à cirurgia e, na seqüência, à radioterapia. Quando a resposta era parcial após 3 ciclos, administravase um quarto e último ciclo, para posterior avaliação das condições de operabilidade. Caso, não ocorresse resposta após 3 ciclos, a QT era suspensa e a paciente encaminhada para tratamento radioterápico.

As respostas pós-QT foram classificadas em: resposta completa - desaparecimento clínico total da neoplasia; resposta parcial - diminuição superior a $50 \%$ do volume tumoral; sem resposta - diminuição inferior a $50 \%$ e resposta global - soma das respostas completas e parciais.

Após 3 a 4 semanas do término da QT, as pacientes que se tornaram operáveis foram submetidas à cirurgia de Wertheim-Meigs. Três a quatro semanas após a cirurgia, as pacientes fo- 
ram encaminhadas ao serviço de radioterapia e submetidas à radioterapia externa, com dose de 5000 cGy, abrangendo toda a pelve. As pacientes que não se tornaram operáveis após a QT neoadjuvante foram encaminhadas para radioterapia, recebendo a dose preconizada para o tratamento radioterápico exclusivo (pélvico externo e radiomoldagem), com dose total no ponto A de 8000 cGy. O tempo total do tratamento oscilou em torno de 8 semanas.

Foi realizado seguimento clínico das pacientes a cada 2 meses durante o primeiro ano; no segundo ano, a cada 3 meses; no terceiro ano, a cada 4 meses; no quarto e quinto anos, a cada 6 meses e, a partir do quinto ano, anualmente. Os exames clínicos e laboratoriais foram realizados e solicitados, conforme os seguintes intervalos: exame geral e ginecológico, a cada consulta; ecografia de abdômen total, radiografia de tórax, creatinina e hemograma, a cada 6 meses, durante os 3 primeiros anos. Após o terceiro ano, os exames foram anuais. A citologia oncótica (Papanicolaou) foi realizada semestralmente nos 3 primeiros anos e após, anualmente.

A análise da taxa acumulada de sobrevida após o uso da QT foi feita pelo método de Tabela de Vida, com intervalo de confiança de $95 \%$, processado por software específico para esse fim (SPSS), baseado nos métodos de Tietze-Lewit. Na análise estatística das Tabelas de Vida, foi empregado o teste de Wilcoxon Geham, para comparação das amostras. Os testes, relativos à sobrevida entre operadas e não operadas após QT, também foram avaliados utilizando-se a análise de variância, por se considerar o delineamento casualizado.

\section{Resultados}

Das 60 pacientes selecionadas, apenas 52 foram avaliadas. Oito pacientes foram excluídas da análise: duas devido a recusa em continuar a QT, pois não toleraram as reações causadas pela medicação, sendo a alopécia a principal razão alegada; três não aceitaram a cirurgia e três perderam seguimento.

De acordo com a Tabela 2, a reação colateral mais freqüente à QT foi a alopécia, que ocorreu em todas as pacientes. A seguir, náusea e vômitos, com 54\%. A única que alcançou grau III de toxicidade foi a alopécia e isto aconteceu em $23 \%$ dos casos.

As complicações cirúrgicas que ocorreram pós-QT neoadjuvante foram: disfunção vesical (29\%), infecção de parede (3\%) e linfedema (3\%).
Tabela 2 - Graus e tipos de toxicidade causada pelo uso da quimioterapia neoadjuvante em pacientes com câncer de colo uterino localmente avançados.

\begin{tabular}{lrrrrrrr}
\hline & \multicolumn{2}{c}{ GI } & \multicolumn{2}{c}{ G2 } & \multicolumn{2}{c}{$\mathbf{G 3}$} & Total \\
Tipos & $\mathbf{n}$ & $\%$ & $\mathbf{n}$ & $\%$ & $\mathbf{n}$ & $\%$ & $\mathbf{n}$ \\
\hline Hematológica & 15 & 29 & 5 & 10 & 0 & 20 \\
Náusea/vômitos & 12 & 23 & 15 & 31 & 0 & 28 \\
Alopécia & 34 & 65 & 6 & 12 & 12 & 23 & 52 \\
Renal & 5 & 10 & 0 & & 0 & 5 \\
Pulmonar & 0 & & 0 & & 0 & \\
Cardíaca & 0 & & 0 & & 0 & \\
Outras & 0 & & 0 & & 0 & \\
\hline
\end{tabular}

Toxicidade das drogas de acordo com a tabela da OMS; G: graus de toxicidade G1, G2 ou G3; n: número pacientes.

Na Tabela 3, observa-se o índice de resposta obtido após o uso da QT neoadjuvante. As respostas globais foram de $80,8 \%$, isto é, 4 em cada 5 pacientes responderam. As respostas completas ocorreram em $11,5 \%$ e as respostas parciais, em $69,2 \%$. Em apenas $19,2 \%$ das pacientes as respostas foram inferior a $50 \%$.

Tabela 3 - Taxa de resposta pós-quimioterapia neoadjuvante, por estádio

\begin{tabular}{|c|c|c|c|c|c|c|}
\hline \multirow[b]{3}{*}{ Resp QT } & \multicolumn{5}{|c|}{ Estadio clínico } & \\
\hline & \multicolumn{2}{|c|}{ IIB } & \multicolumn{2}{|c|}{ IIIB } & \multicolumn{2}{|c|}{ Total } \\
\hline & $n$ & $\%$ & $n$ & $\%$ & $n$ & $\%$ \\
\hline $\mathrm{RC}$ & 3 & 11 & 3 & 12 & 6 & 11,53 \\
\hline $\mathrm{RP}$ & 24 & 89 & 12 & 48 & 36 & 69,23 \\
\hline SR & 0 & 0 & 10 & 40 & 10 & 19,24 \\
\hline Total & 27 & 100 & 25 & 100 & 52 & 100,00 \\
\hline
\end{tabular}

Resp. QT: resposta à quimioterapia; $n$ : número de pacientes; RC: resposta completa; RP: resposta parcial; SR: sem resposta.

$\mathrm{O}$ índice de respostas parciais à QT neoadjuvante no estádio IIB foi maior (89\%) do que no estádio IIIB (48\%). As respostas completas ocorreram igualmente nos 2 grupos (no estádio IIB, $11 \%$, e no estádio IIIB, 12\%). Todas as 27 pacientes no estádio IIB tiveram resposta global, enquanto que, entre as 25 pacientes do estádio IIIB, isto ocorreu em 15 pacientes $(60 \%)$.

Após a QT neoadjuvante, 2 em cada 3 pacientes do grupo de estudo tornaram-se operáveis $(65,5 \%)$. Foram operadas quase o triplo de pacientes em estádio inicial IIB, em relação àquelas cujo estádio inicial era IIIB (93 versus 36\%). Entre as 27 pacientes do estádio IIB, quase a totalidade (93\%) tornou-se operável pós-QT neoadjuvante, enquanto apenas 9 pacientes (36\%) no estádio IIIB foram submetidas à cirurgia preconizada (Tabela 4). 
Tabela 4 - Taxa de operabilidade pós-quimioterapia neoadjuvante, conforme estádio clínico prévio ao tratamento.

\begin{tabular}{|c|c|c|c|c|c|c|}
\hline \multirow[b]{3}{*}{ Cirurgia } & \multicolumn{4}{|c|}{ Estadio clínico } & & \\
\hline & \multicolumn{2}{|c|}{ IIB } & \multicolumn{2}{|c|}{ IIIB } & \multicolumn{2}{|c|}{ Total } \\
\hline & $\mathrm{n}$ & $\%$ & $n$ & $\%$ & $\mathrm{n}$ & $\%$ \\
\hline Operadas & 25 & 93 & 9 & 36 & 34 & 65,5 \\
\hline Não operadas & 2 & 7 & 16 & 64 & 18 & 33,5 \\
\hline Total & 27 & 100 & 25 & 100 & 52 & 100 \\
\hline
\end{tabular}

$\mathrm{n}$ : número de pacientes.

A sobrevida em todo o grupo, após a QT neoadjuvante, em um seguimento de cinco anos, foi de $62,61 \%$. Na análise da sobrevida por estadiamento, notou-se sobrevida maior nas pacientes no estádio IIB $(77 \%)$ em relação às pacientes IIIB $(45 \%)$, com $\mathrm{p}=0,02$, diferença esta demonstrada na Figura 1.

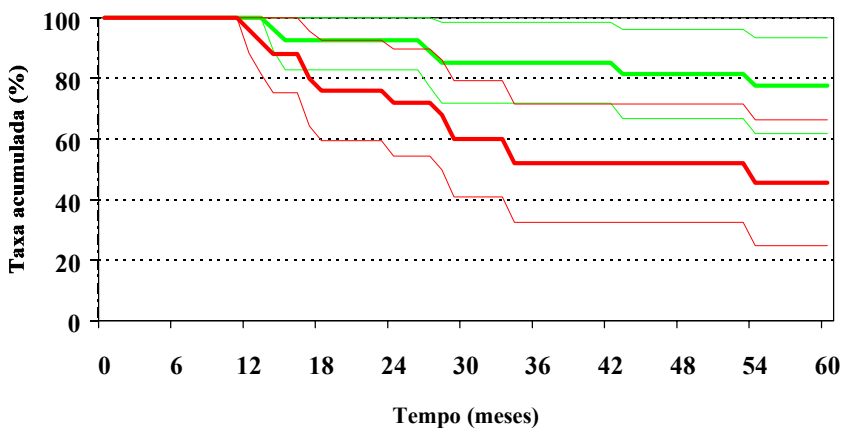

Curva 1: taxa acumulada de sobrevida em 5 anos no estádio IIB; curva 2: taxa acumulada de sobrevida em 5 anos no estádio IIIB.

Figura 1 - Taxa acumulada de sobrevida pós-quimioterapia neoadjuvante, segundo 0 estádio inicial, no câncer do colo uterino.

O estudo das curvas de sobrevida, com intervalo mensal para o total da amostra, evidencia que entre as pacientes que foram operadas pósQT neoadjuvante, a taxa de sobrevida em 5 anos foi 4 vezes maior em relação àquelas pacientes que não foram operadas $(82,14$ versus $16,67 \%$, respectivamente). Essas diferenças, segundo o teste de Wilcoxon Geham, têm significado estatístico $(\mathrm{p}<0,01)$. Das 52 pacientes avaliadas, $34(65,5 \%)$ foram operadas e $18(33,5 \%)$ não puderam ser submetidas ao tratamento cirúrgico.

Ao mesmo tempo que a sobrevida global em cinco anos foi quase duas vezes maior no estádio IIB em relação ao estádio IIIB não houve diferença de sobrevida entre os estádios IIB e IIIB, quando se comparam somente as pacientes que foram operadas. Em ambos os estádios, quando operadas, as pacientes alcançaram sobrevida global em cinco anos de aproximadamente $80 \%$. O p de 0,70 ao se compararem os casos operados, IIB e IIIB, significa que, com base nessa amostra, não há evi- dências para rejeitar a hipótese de que as taxas de sobrevida sejam iguais.

Os dados relativos à sobrevida, obtidos pela análise de variância, entre pacientes submetidas ou não à cirurgia pós-QT neoadjuvante, mostram diferença estatística significante, mesmo com alto coeficiente de variação, fato de essencial reforço ao resultado encontrado.

\section{Discussão}

Neste ensaio clínico, o tratamento pela QT neoadjuvante revelou-se método com boa tolerabilidade e aceitabilidade por parte das pacientes. Este resultado está de acordo com a literatura, na qual vários autores têm confirmado a tolerabilidade da QT para tratamento de câncer do colo uterino ${ }^{2,5-9}$.

É de grande relevância e pertinência, considerando os casos aqui avaliados, relatar que não ocorreu óbito e também foi desnecessário suspender o esquema de QT. Também não ocorreu nenhum efeito tóxico após QT neoadjuvante que colocasse em risco a vida da paciente. Ainda sobre a baixa toxicidade da QT, afirme-se que tem sido descrita por vários autores ${ }^{2,7-9}$.

Sempre houve dúvidas se o tratamento pela QT neoadjuvante é capaz de aumentar o índice de complicações cirúrgicas, principalmente em razão da ação destes fármacos em diminuir a imunidade e a este fator somar-se outro, o possivel efeito de retardar a cicatrização. Muito embora e apesar de ser grande o porte cirúrgico do procedimento realizado (Classe III na Classificação das Histerectomias de Piver e Rutledge ${ }^{10}$, foi pouco significativo o índice de complicações cirúrgicas ocorrido com as pacientes deste estudo. Ressaltese que este índice obtido encontra-se dentro dos limites inferiores, descritos na literatura, quando o tratamento realizado é exclusivamente cirúrgico $^{11}$. Acrescente-se que também não houve maior dificuldade técnica para a realização do ato cirúrgico pós-QT neoadjuvante.

No grupo das pacientes observadas, o tratamento pela QT neoadjuvante mostrou-se eficaz em obter regressão dos tumores. Também se observou que todas as pacientes do estádio IIB obtiveram resposta, ao passo que no estádio IIIB, houve apenas $60 \%$ de respostas. Estes resultados parecem confirmar os resultados da literatura, que mostram que quanto maior for o volume tumoral, menor é a eficácia do tratamento pela QT. Para efeito de clareza, infere-se aqui que este nivel elevado de respostas obtém-se apenas quando a quimioterapia é usada como tratamento primário (anterior a outros tratamentos). Na literatura, cons- 
ta que as pacientes com tratamento prévio (cirurgia e/ou radioterapia) apresentam nivel de resposta à quimioterapia inferior em duas a três vezes em relação àquelas que não foram tratadas ${ }^{12,13}$. A explicação para tal fato se deve a diminuição da vascularização tumoral (pelos procedimentos anteriores), impedindo assim o aporte ideal do quimioterápico ao tumor.

No grupo de pacientes estudadas, o tratamento pela QT neoadjuvante mostrou-se eficaz, não apenas em obter a regressão das lesões, mas também em tornar operáveis tumores antes considerados inoperáveis. Há grande pertinência no fato de o tratamento pela QT neoadjuvante permitir que $65 \%$ das pacientes de todo este grupo fossem submetidas à cirurgia. Estes resultados também foram relatados por vários autores ${ }^{7,8,14-16}$.

A sobrevida global em 5 anos de todo o grupo foi de $62,6 \%$, cerca de 12 pontos percentuais acima da média da literatura em pacientes IIB e IIIB submetidas ao tratamento radioterápico exclusivo $^{4,17}$. Quando se avaliou a sobrevida global, separadamente por estádio, observou-se que no grupo IIB houve 17 pontos percentuais acima da média histórica e no grupo IIIB, apenas 5 pontos percentuais acima. Estes dados poderiam sugerir que pela QT neoadjuvante haveria melhor sobrevida no estádio IIB. Entretanto, quando se comparou o grupo de pacientes operadas com o grupo de não operadas, os resultados de sobrevida global de $80 \%$ em 5 anos foram idênticos aos obtidos nos estádios iniciais (I e IIA). Por outro lado, quando se analisou o grupo de pacientes não operadas, encontrou-se taxa de sobrevida global de $16,6 \%$, valor este inferior aos existentes na literatura com pacientes submetidas ao tratamento radioterápico exclusivo. Provável explicação para este fato é que a QT neoadjuvante poderia selecionar clones de células resistentes à radioterapia ${ }^{2,7}$, ou que pode existir resistência cruzada, entre o tratamento quimioterápico e o radioterápico ${ }^{18}$.

A seqüência QT-cirurgia-radioterapia, teoricamente, permite melhor controle local e sistêmico do câncer do colo uterino localmente avançado. O controle local mais adequado obtémse pela retirada cirúrgica dos clones resistentes, somados aos efeitos da radioterapia pélvica, que eliminam as possiveis margens cirúrgicas microscópicas comprometidas, com doses variando de 4500 e $5000 \mathrm{cGy}^{4}$. A radioterapia e a cirurgia são tratamentos locais, porém, com mecanismos de ação diferentes, e as duas modalidades terapêuticas se complementam. O melhor controle sistêmico ocorre pela possibilidade de a QT agir nos eventuais focos de micrometástases e diminuir as chances de aparecimento de metástases a distância ${ }^{19}$.

Havia dúvidas sobre a eficiência da QT no tratamento do câncer do colo. A partir dos resultados de quatro estudos prospectivos e randomizados $^{20,21}$, utilizando QT concomitante com radioterapia, evidenciou-se que existe definitivamente lugar de destaque para a QT no tratamento dos tumores do colo uterino. Este trabalho confirmou que esse tumor é químio-sensivel, conseguindose operar dois terços das pacientes que eram consideradas inoperáveis antes da QT, e que as operadas alcançaram uma sobrevida global em 5 anos de $80 \%$. Apesar de não ser um trabalho analitico, os seus dados sugerem que o tratamento seqüencial QT-cirurgia-radioterapia possa ser modalidade terapêutica viável a ser oferecida às pacientes com câncer do colo uterino localmente avançados.

\section{ABSTRACT}

Purpose: to evaluate neoadjuvant chemotherapy in locally advanced cervical cancer as to its acceptability, tolerability, toxicity, surgical complications, operability, response rate, and overall survival in 5 years.

Methods: sixty women with locally advanced cervical cancer (stages IIB and IIIB), who were submitted to neoadjuvant chemotherapy, were included. All patients were treated with doxorubicin-bleomycin-cisplatin. Those who had a good response, allowing a surgical approach, underwent the Wertheim-Meigs procedure. After surgery, they were submitted to pelvic radiotherapy. Those that could not be submitted to surgery after chemotherapy underwent total radiotherapy. Results: the average follow-up was 108 months, and $80 \%$ of the patients had an overall response to neoadjuvant chemotherapy. In the IIB group, the response rate was $100 \%$, and in the IIIB group it was $60 \%$. The operability rate after neoadjuvant chemotherapy was $65 \%$. The overall survival in 5 years was $62 \%$. Comparing the operated group $(n=34)$ with the nonoperated group $(n=18)$, the overall survival in 5 years was 82.14 and $16.67 \%$, respectively.

Conclusions: neoadjuvant chemotherapy with doxorubicinbleomycin-cisplatin for locally advanced cervical cancer is safe, with a low rate of side effects, and allowed a high operability rate.

KEY WORDS: Cervical cancer. Neoadjuvant chemotherapy. Cancer: relapse.

\section{Referências}

1. Rose PG, Adler LP, Rodrigues M, Faulhaber PF, AbdulKarin FW, Miraldi F. Positron emission tomography for evaluating para-aortic nodal metastasis in locally advanced cervical cancer before surgical staging: a surgicopathologic study. J Clin Oncol 1999; 17:41-5. 
2. Greven K, Petereit D, Vermorken JB, Lanciano R. Current developments in the treatment of newly diagnosed cervical cancer. Hematol Oncol Clin North Am 1999; 13:275-303.

3. Keys H, Gibbons SK. Optimal management of locally advanced cervical carcinoma. J Natl Cancer Inst Monogr 1996; (21):89-92.

4. Schünemann Júnior E. Avaliação preliminar da quimioterapia neoadjuvante no câncer avançado do colo de útero. Temas livres do $11^{\circ}$ Congresso Brasileiro de Cancerologia; 1987; Florianópolis, Brasil.

5. Sardi JE, Giaroli A, Sananes C, et al. Long-term follow-up of the first randomized trial using neoadjuvant chemotherapy in stage IB squamous carcinoma of the cervix: the final results. Gynecol Oncol 1997; 67:61-9.

6. Benedetti-Panici P, Greggi S, Scambia G, et al. Longterm following neoadjuvant chemotherapy and radical surgery in locally advanced cervical cancer. Eur J Cancer 1998; 34:341-6.

7. Murad AM, Triginelli AS, Tamilini W, et al. Phase II multicentric trial of neoadjuvant Bleomycin, Ifosfamide and Carboplatin (BIC) in locally advanced cervical cancer. Oncol Atual 1998; 8:210.

8. DiSaia PJ. The case against the surgical concept of en bloc dissection for certain malignancies of the reproductive tract. Cancer 1987; 60 (8 Suppl):202534.

9. Magrina JF. Primary surgery for stage IB-IIA cervical cancer, including short-term and long-term morbidity and treatment in pregnancy. J Natl Cancer Inst Monogr 1996; (21):53-9.

10.Lacava JA, Leone BA, Machiavelli M, et al. Vinorelbine as neoadjuvante chemotherapy in advanced cervical carcinoma. J Clin Oncol 1997; 15:604-9.

11.Friedlander M, Kaye SB, Sullivan A, et al. Cervical carcinoma: a drug responsive tumor - experience with combined cisplatin, vinblastine, and bleomycin therapy. Gynecol Oncol 1983; 16:275-81.
12.Chang $\mathrm{HC}$, Lai $\mathrm{CH}$, Chou PC, et al. Neoadjuvant chemotherapy with cisplatin, vincristine, and bleomycin and radical surgery in early-stage bulky cervical carcinoma. Cancer Chemother Pharmacol 1992; 30:281-5.

13.Eddy GL. Neoadjuvant chemotherapy before surgery in cervical cancer. J Natl Cancer Inst Monogr 1996; (21):93-9.

14.Schünemann Júnior E, Mioshi CK, Soares EWS, Catani GSA. Quimioterapia neo-adjuvante no câncer de colo uterino. Acta Oncol Bras 1997; 17:7-11.

15.Kavanagh JJ, Verschraegen CF, Kudelka AP. Irinotecan in cervical cancer. Oncology (Huntingt) 1998; 12 (8 Suppl 6):94-8.

16.Colombo A, Landoni F, Maneo A, Zanetta G, Nava S, Tancini G. Neoadjuvant chemotherapy to radiation and concurrent chemoradiation for locally advanced squamous cell carcinoma of the cervix: a review of the recent literature. Tumori 1998; 84:22937.

17.Salum R, Lopes ER, Souza MAH. Radiumterapia préoperatória e histerectomia radical no tratamento do câncer do colo uterino IB, IIA e IIB inicial. Rev Bras Ginecol Obstet 1995; 17:635-60.

18. Whitney CW, Sause W, Bundy BN, et al. Randomized comparison of fluorouracil plus cisplatin versus hidroxyurea as an adjunct to radiation therapy in stage IIB - IVA carcinoma of the cervix with negative para-aortic lymph nodes: a Gynecologic Oncology Group and Southwest Oncology Group study. J Clin Oncol 1999; 17:1339-48.

19.Keys HM, Bundy BN, Stehman FB, et al. Cisplatin, radiation, and adjuvant hysterectomy compared with radiation and adjuvant hysterectomy for bulky stage IB cervical carcinoma. N Engl J Med 1999; 340:115561.

20.Rose PG, Bundy BN, Watkins EB, et al. Concurrent cisplatin-based radiotherapy and chemotherapy for locally advanced cervical cancer. N Engl J Med 1999; 340:1144-53.

21.Morris M, Eifel PJ, Lu J, et al. Pelvic radiation with concurrent chemotherapy compared with pelvic and para-aortic radiation for high-risk cervical cancer. N Engl J Med 1999; 340:1137-43. 